\title{
MUTAÇÕES NO GENE CYP21A2 ANALISADAS POR MEIO DA REAÇÃO DA POLIMERASE EM CADEIA
}

\author{
MUTATIONS IN THE CYP21A2 GENE ANALYZED BY THE POLYMERASE CHAIN \\ REACTION
}

\section{MUTACIONES EN EL GEN CYP21A2 ANALIZADAS A TRAVÉS DE LA REACCIÓN DE LA POLIMERASA EN CADENA}

Carlos Virgílio Rocha de Sousa Silva ${ }^{1}$, Gustavo Maffra Monteiro², Aluísio Antônio Brito de Mesquita $^{3}$, Susane Vasconcelos Zanotti ${ }^{4}$, Isabella Lopes Monlleó, Reginaldo José Petroli ${ }^{5}$

\section{RESUMO}

Objetivo: padronizar a Reação em Cadeia da Polimerase Alelo Específico (PCR-AS), no Laboratório de Genética Molecular Humana do Hospital Universitário Professor Alberto Antunes (HUPAA), para a investigação das mutações p.Pro30Leu, c.290-13A/C>G, p.Gly110fs, p.Ile172Asn, Cluster6 (p.Ile236Asn+p.Val237Glu+p.Met239Lys), p.Val281Leu, p.Gln318* e p.Arg356Trp no gene CYP21A2, todas de reconhecida frequência e provenientes do pseudogene CYP21A1P. Método: as PCR-AS foram realizadas utilizando amostras-controle para cada mutação. Oligonucleotídeos para a seleção do alelo, com a alteração (mutante) e sem a alteração (normal), foram utilizados para cada mutação analisada. Os produtos das PCR-AS foram testados por meio de eletroforese em gel de agarose. A análise dos resultados das reações foi realizada por meio da presença ou ausência do fragmento de interesse. Resultados: os alelos normal e mutante foram amplificados para as mutações p.Pro30Leu, c.290-13A/C>G, p.Gly110fs, p.Ile172Asn, Cluster6 (p.Ile236Asn+p.Val237Glu+p.Met239Lys), p.Val281Leu, p.Gln318* e p.Arg356Trp. Conclusão: a padronização PCR-AS para o diagnóstico molecular de HAC devido às mutações no gene CYP21A2, no Laboratório de Genética Molecular Humana, proporcionará a elucidação diagnóstica de casos triados pelo Programa Nacional de Triagem Neonatal e/ou atendidos no Serviço de Genética Clínica do HUPAA/UFAL. Além disso, proporcionará o reconhecimento das mutações mais frequentes no gene CYP21A2 na população de Alagoas, assim como a ampliação do conhecimento sobre as relações genótipo-fenótipo para os casos de HAC. Este conhecimento é de fundamental importância para incrementar a abordagem diagnóstica, o tratamento, o aconselhamento genético e a prevenção.

\section{Descritores: Reação em Cadeia da Polimerase; Genética Médica; Hiperplasia.}

\footnotetext{
${ }^{1}$ Biólogo. Universidade Federal de Alagoas/UFAL. Maceió (AL), Brasil.

2 Graduando de Licenciatura em Ciências Biológicas. Universidade Federal de Alagoas/UFAL. Maceió $(A L)$, Brasil.

3 Graduando em Medicina. Universidade Federal de Alagoas/UFAL. Maceió (AL), Brasil.

4,5,6 Doutores. Universidade Federal de Alagoas/UFAL. Maceió (AL), Brasil.
}

Rev. Port. Saúde e Sociedade. 2019;4(1): 985 - 993. 


\section{ABSTRACT}

Objective: to standardize the Allele-Specific Polymerase Chain Reaction (PCR-AS) in the Laboratory of Human Molecular Genetics of the Professor Alberto Antunes University Hospital (HUPAA) to investigate the mutations p.Pro30Leu, c.290-13A / C> G, p .Gly110fs, p.Il172Asn, Cluster6 (p.Ile236Asn + p.Val237Glu + p.Met239Lys), p.Val281Leu, p.Gln318 * and p.Arg356Trp in the CYP21A2 gene, all of recognized frequency and from the pseudogene CYP21A1P. Method: PCR-AS were performed using control samples for each mutation. Oligonucleotides for allele selection, with alteration (mutant) and without alteration (normal), were used for each mutation analyzed. PCR-AS products were tested using agarose gel electrophoresis. The analysis of reaction results was performed through the presence or absence of the fragment of interest. Results: the normal and mutant alleles were amplified for the mutations p.Pro30Leu, c.290-13A / C> G, p.Gly110fs, pIl172Asn, Cluster6 (p.Ile236Asn + p.Val237Glu + p.Met239Lys), p .Val281Leu, p.GIn318 * and p.Arg356Trp. Conclusion: the PCR-AS standardization for the molecular diagnosis of CAH due to mutations in the CYP21A2 gene in the Laboratory of Human Molecular Genetics will provide the diagnostic elucidation of cases screened by the National Neonatal Screening Program and / or attended at the Clinical Genetic Service of HUPAA / UFAL. In addition, it will provide recognition of the most frequent mutations in the CYP21A2 gene in the population of Alagoas, as well as the increase of knowledge about genotype-phenotype relationships for cases of $\mathrm{CAH}$. This knowledge is of fundamental importance to increase the diagnostic approach, treatment, genetic counseling and prevention.

\section{Descriptors: Polymerase Chain Reaction; Genetics, Medical; Hyperplasia.}

\section{RESUMEN}

Objetivo: estandarizar la Reacción en Cadena de la Polimerasa Alelo Específico (PCR-AS), en el laboratorio de Genética Molecular Humana del Hospital Universitario Profesor Alberto Antunes (HUPAA), para la investigación de las mutaciones p.Pro30Leu, c.290-13a /C>G, p.Gly110fs, p.Ile172Asn, Cluster6 (p.Ile236Asn+p.Val237Glu+p.Met239Lys), p.Val281Leu, p.GIn318* y p.Arg356Trp en el gen CYP21A2, todas de reconocida frecuencia y provenientes del pseudogén CYP21A1P. Método: las PCR-AS se realizaron utilizando muestras de control para cada mutación. Se utilizaron los Oligonucleótidos para la selección del alelo, con la alteración (mutante) y sin la alteración (normal), para cada mutación analizada. Los productos de las PCR-AS fueron probados a través de electroforesis en gel de agarosa. El análisis de los resultados de las reacciones se realizó a través de la presencia o ausencia del fragmento de interés. Resultados: Los alelos normal y mutante se han amplificado para las mutaciones p.Pro30Leu, c.290-13A / C> G, p.Gly110fs, p.Ile172Asn, Cluster6 (p.Ile236Asn + p.Val237Glu + p.Met239Lys), p .Val281Leu, p.Gln318* y p.Arg356Trp. Conclusión: la estandarización PCR-AS para el diagnóstico molecular de HAC debido a las mutaciones en el gen CYP21A2, en el Laboratorio de Genética Molecular Humana, proporcionará la elucidación diagnóstica de casos triados por el Programa Nacional de Triaje Neonatal y / o atendidos en el Servicio de Genética Clínica del HUPAA / UFAL. Además, proporcionará el reconocimiento de las mutaciones más frecuentes en el gen CYP21A2 en la población de Alagoas, así como la ampliación del conocimiento sobre las relaciones genotipo-fenotipo para los casos de HAC. Este conocimiento es de fundamental importancia para incrementar el abordaje diagnóstico, el tratamiento, el asesoramiento genético y la prevención.

Descriptores: Reacción en Cadena de la Polimerasa; Genética Médica; Hiperplasia.

Rev. Port. Saúde e Sociedade. 2019;4(1): 985 - 993. 


\section{INTRODUÇÃO}

Os distúrbios da diferenciação do sexo (DDS) são condições congênitas onde o desenvolvimento genital ou gonadal é incompleto ou desordenado, levando a uma discordância entre o sexo genético, gonadal e fenotípico do indivíduo afetado. ${ }^{1}$ Entre os inúmeros casos desses distúrbios, encontra-se a hiperplasia adrenal congênita (HAC), um erro inato do metabolismo caracterizado pela deficiência de uma das cinco enzimas envolvidas na esteroidogênese adrenal, a partir do colesterol. Cerca de $90 \%$ a $95 \%$ dos casos ocorrem devido à deficiência da enzima CYP21A2, codificada pelo gene CYP21A2 (ENSG00000231852).2-3

As manifestações clínicas associadas à HAC são divididas em duas formas: a forma clássica, com os fenótipos perdedor de sal (PS) e virilizante simples (VS), e a forma não clássica ou de início tardio, que inclui os subgrupos sintomáticos e assintomáticos. ${ }^{1-3}$

O espectro clínico da HAC é amplo e pode variar de acordo com a atividade residual da enzima CYP21A2 alterada. A forma VS ocorre devido à atividade residual enzimática calculada entre $1 \%$ e $5 \%$, tendo, como resultado, apenas a deficiência isolada da produção de cortisol, sem prejuízos na produção de aldosterona. Já a forma PS ocorre quando a atividade residual enzimática é nula ou inferior a $1 \%$, resultando na deficiência da biossíntese de cortisol e de aldosterona, com o desvio da rota para a produção de andrógenos. ${ }^{3}$

Quando não tratados, os sinais clínicos do hiperandrogenismo podem ser observados em todas as faixas etárias, desde o nascimento até a idade adulta. Esses sinais compreendem a ambiguidade genital de diferentes graus em sujeitos $46, X X$, a puberdade precoce, o hirsutismo, a amenorreia/oligomenorreia e a infertilidade. Na forma perdedora de sal, que corresponde a $75 \%$ dos casos, a deficiência de cortisol e mineralocorticoides pode estar associada a alterações hidroeletrolíticas, como a desidratação (hiponatrêmica e hipercalêmica), os vômitos, a acidose metabólica e o choque hipovolêmico, com um significativo aumento da mortalidade, especialmente, no período neonatal. ${ }^{4-5}$

A forma não clássica sintomática é caracterizada por não apresentar a virilização pré-natal, e os sintomas se desenvolvem em épocas variáveis. Mulheres jovens podem apresentar acne, hirsutismo e desordens menstruais, e as crianças afetadas caracterizam-se por apresentar a puberdade precoce, a velocidade de crescimento acelerada e a maturação esquelética avançada. ${ }^{2-3,6-7} \mathrm{~A} H \mathrm{HC}$, na forma

Rev. Port. Saúde e Sociedade. 2019;4(1): 985 - 993. 
não clássica assintomática, apresenta um perfil hormonal semelhante à forma sintomática, porém, não desenvolve manifestações clínicas. ${ }^{1,8-9}$

A HAC apresenta uma prevalência global de $1: 10.000$ a 1:15.000 nascidos vivos. $^{4,5}$ Dados de triagem neonatal em alguns Estados brasileiros indicam incidências de 1:10.325 em Goiás; ${ }^{10}$ 1:19.927 em Minas Gerais ${ }^{11}$ e 1:11.655 em Santa Catarina, ${ }^{12}$ porém, ainda não há um dado único e preciso que possa informar a incidência da HAC no Brasil.

Segundo "The Human Gene Mutation Database" (2018), um total de 302 mutações foi descrito no CYP21A2 relacionado à HAC. ${ }^{14}$ Dessas, oito mutações, derivadas do pseudogene $C Y P 21 A 1 P$, são encontradas com maior frequência nos indivíduos afetados: p.Pro30Leu; c.290-13A/C>G; p.Gly110fs; p.Ile172Asn; Cluster6 (p.Ile236Asn+p.Val237Glu+p.Met239Lys); p.Val281Leu; p.GIn318* e p.Arg356Trp.

O rastreamento das mutações frequentes no $C Y P 21 A 2$ pode ser realizado pela reação em cadeia da polimerase alelo específica (PCR-AS), cujos dados moleculares são de grande importância para a definição do diagnóstico. Com base no exposto, o objetivo deste trabalho foi padronizar a PCR-AS no Laboratório de Genética Molecular Humana do Hospital Universitário Professor Alberto Antunes da Universidade Federal de Alagoas (LGMH/HUPAA/UFAL) para a investigação das mutações p.Pro30Leu, c.290-13A/C>G, p.Gly110fs, p.Ile172Asn, Cluster6 (p.Ile236Asn+p.Val237Glu+p.Met239Lys)， p.Val281Leu， p.Gln318* e p.Arg356Trp, todas de reconhecida frequência em sujeitos com HAC.

\section{MÉTODOS}

Os experimentos foram realizados no LGMH/HUPAA/UFAL. A verba para a execução do projeto foi proveniente do projeto Universal - FAPEAL, intitulado Caracterização Molecular de Famílias com Hiperplasia Adrenal Congênita em Alagoas: Estudo Piloto (EDITAL UNIVERSAL FAPEAL No 04/2016 - PROCESSO No 60030001071/2016).

Este trabalho foi conduzido sob a aprovação do Comitê de Ética em Pesquisa da Universidade Federal de Alagoas (CAAE: 59931616.6.0000.5013 - Parecer 1.752.433 de 29/09/2016).

O DNA genômico de pacientes previamente estudados serviu como o controle das reações. Os controles foram gentilmente cedidos pela Dra. Maricilda

Rev. Port. Saúde e Sociedade. 2019;4(1): 985 - 993. 
Palandi de Mello, do Centro de Biologia Molecular e Engenharia Genética da Universidade Estatual de Campinas. As mutações analisadas foram p.Pro30Leu, c.290-13A/C>G, p.Gly110fs, p.Ile172Asn, Cluster6 (p.Ile236Asn+p.Val237Glu+p.Met239Lys)， p.Val281Leu， p.Gln318* e p.Arg356Trp.

Para a identificação dos alelos normais e mutantes, foram realizadas duas PCRs. Em uma reação, foram utilizados o oligonucleotídeo normal e o oligonucleotídeo âncora e, na outra reação, o oligonucleotídeo mutante e o âncora, sendo que ambas foram realizadas em tubos diferentes. Devido à grande homologia entre o gene e o pseudogene, o oligonucleotídeo âncora é fundamental para a seleção e a amplificação exclusiva do gene CYP21A2.

Os fragmentos amplificados foram analisados por meio da eletroforese em gel de agarose a $1 \%$, com o auxílio da régua de peso molecular $1 \mathrm{~kb}$ plus DNA Ladder.

Os géis foram corados em uma solução de brometo de etídio $(0,5 \mu \mathrm{g} / \mathrm{mL})$ e os resultados foram analisados em um transluminador, onde foram capturadas as fotos dos géis. A análise dos resultados foi realizada por meio da constatação da presença ou ausência do fragmento de interesse.

\section{RESULTADOS}

A amplificação dos fragmentos de interesse, por meio da PCR-AS, foi alcançada para as mutações p.Pro30Leu, c.290-13A/C>G, p.Gly110fs, p.Ile172Asn, Cluster6 (p.Ile236Asn+p.Val237Glu+p.Met239Lys), p.Val281Leu, p.GIn318* e p.Arg356Trp.

\begin{tabular}{lccccccccc}
\hline $\begin{array}{l}\text { Reagentes } \\
\text { (microlitros) }\end{array}$ & $\begin{array}{c}\text { p.Pro30Le } \\
\mathbf{u} * * *\end{array}$ & $\begin{array}{c}\text { SPLC C } \\
\text { e G* }\end{array}$ & SPLC A & $\begin{array}{c}\text { p.Gly1 } \\
\text { 10fs* }\end{array}$ & $\begin{array}{c}\text { p.II172A } \\
\mathbf{S n} \\
* *\end{array}$ & $\begin{array}{c}\text { Cluster6 } \\
*\end{array}$ & $\begin{array}{c}\text { p.Val281Le } \\
\mathbf{u * * *}\end{array}$ & $\begin{array}{c}\text { p.GIn318 } \\
*\end{array}$ & $\begin{array}{c}\text { p.Arg356 } \\
\text { Trp* }\end{array}$ \\
\hline Água & 17 & 20 & 19,9 & 20,1 & 20,3 & 20,1 & 19,5 & 20 & 20,3 \\
Tampão & 6 & 3 & 3 & 3 & 3 & 3 & 3 & 3 & 3 \\
MgCl & 1 & 0,7 & 0,8 & 0,9 & 0,7 & 0,9 & 1 & 1 & 0,7 \\
DNTP & 2,5 & 2,5 & 2,5 & 2,5 & 2,5 & 2,5 & 3 & 2,5 & 2,5 \\
Primer 1 & 1 & 1 & 1 & 1 & 1 & 1 & 1 & 1 & 1 \\
Primer 2 & 1 & 1 & 1 & 1 & 1 & 1 & 1 & 1 & 1 \\
BSA 1\% & 0,3 & 0,3 & 0,3 & 0,3 & 0,3 & 0,3 & 0,3 & 0,3 & 0,3 \\
Taq & 0,2 & 0,3 & 0,5 & 0,2 & 0,2 & 0,2 & 0,2 & 0,2 & 0,2 \\
\hline
\end{tabular}

Tabela 01 - Reagentes utilizados em cada reação.

Forma: *Perdedora de sal; **Virilizante simples; ***Não clássica

Rev. Port. Saúde e Sociedade. 2019;4(1): 985 - 993. 


\begin{tabular}{|c|c|c|c|c|c|c|c|c|c|}
\hline & $\begin{array}{l}\text { p.Pro30 } \\
\text { Leu*** }\end{array}$ & $\begin{array}{l}\text { SPLC A } \\
\text { e G }\end{array}$ & $\begin{array}{c}\text { SPLC } \\
\text { C }\end{array}$ & $\begin{array}{c}\text { P.Gly11 } \\
\text { Ofs* }\end{array}$ & $\begin{array}{c}\text { p.II172As } \\
\text { n } \\
* *\end{array}$ & $\underset{*}{\text { Cluster6 }}$ & $\begin{array}{c}\text { p.Val281L } \\
\text { eu*** }\end{array}$ & $\underset{*}{\text { p.Gln318 }}$ & $\begin{array}{c}\text { p.Arg356Tr } \\
p^{*}\end{array}$ \\
\hline \multirow[t]{9}{*}{ Ciclos } & $\begin{array}{c}94^{\circ} \mathrm{C}-5 \\
\text { min }\end{array}$ & $\begin{array}{c}94^{\circ} \mathrm{C}- \\
5 \mathrm{~min}\end{array}$ & $\begin{array}{c}94{ }^{\circ} \mathrm{C} \\
-5 \\
\text { min }\end{array}$ & $\begin{array}{l}94^{\circ} \mathrm{C}- \\
5 \mathrm{~min}\end{array}$ & $\begin{array}{c}94^{\circ} \mathrm{C}-5 \\
\mathrm{~min}\end{array}$ & $\begin{array}{l}94^{\circ} \mathrm{C}- \\
5 \mathrm{~min}\end{array}$ & $\begin{array}{c}95^{\circ} \mathrm{C}-5 \\
\min \end{array}$ & $\begin{array}{c}94^{\circ} \mathrm{C}-5 \\
\mathrm{~min}\end{array}$ & $\begin{array}{c}94^{\circ} \mathrm{C}-5 \\
\mathrm{~min}\end{array}$ \\
\hline & $\begin{array}{c}94^{\circ} \mathrm{C}-1 \\
\min \end{array}$ & $\begin{array}{l}94^{\circ} \mathrm{C}- \\
1 \mathrm{~min}\end{array}$ & $\begin{array}{c}94^{\circ} \mathrm{C} \\
-1 \\
\text { min }\end{array}$ & $\begin{array}{l}94^{\circ} \mathrm{C}- \\
1 \mathrm{~min}\end{array}$ & $\begin{array}{c}94^{\circ} \mathrm{C}-1 \\
\min \end{array}$ & $\begin{array}{l}94^{\circ} \mathrm{C}- \\
1 \mathrm{~min}\end{array}$ & $\begin{array}{c}95^{\circ} \mathrm{C}-1 \\
\min \end{array}$ & $\begin{array}{c}94^{\circ} \mathrm{C}-1 \\
\min \end{array}$ & $\begin{array}{c}94^{\circ} \mathrm{C}-1 \\
\min \end{array}$ \\
\hline & $\begin{array}{c}67^{\circ} \mathrm{C}-1 \\
\mathrm{~min}\end{array}$ & $\begin{array}{l}67,5 \circ \mathrm{C} \\
-1 \mathrm{~min}\end{array}$ & $\begin{array}{c}67{ }^{\circ} \mathrm{C} \\
-1 \\
\text { min }\end{array}$ & $\begin{array}{l}67,5 \circ \mathrm{C} \\
-1 \mathrm{~min}\end{array}$ & $\begin{array}{c}67^{\circ} \mathrm{C}-1 \\
\mathrm{~min}\end{array}$ & $\begin{array}{l}67,5{ }^{\circ} \mathrm{C} \\
-1 \mathrm{~min}\end{array}$ & $\begin{array}{c}61{ }^{\circ} \mathrm{C}-1 \\
\mathrm{~min}\end{array}$ & $\begin{array}{c}58^{\circ} \mathrm{C}-1 \\
\min \end{array}$ & $\begin{array}{c}62^{\circ} \mathrm{C}-1 \\
\min \end{array}$ \\
\hline & $\begin{array}{c}72^{\circ} \mathrm{C}- \\
2 \mathrm{~min} 30 \\
\text { seg }\end{array}$ & $\begin{array}{l}72^{\circ} \mathrm{C}- \\
1 \mathrm{~min}\end{array}$ & $\begin{array}{c}72^{\circ} \mathrm{C}- \\
2 \mathrm{~min} \\
30 \\
\text { seg }\end{array}$ & $\begin{array}{c}72^{\circ} \mathrm{C}-1 \\
\min \end{array}$ & $\begin{array}{c}72^{\circ} \mathrm{C}-10 \\
\min \end{array}$ & $\begin{array}{c}72^{\circ} \mathrm{C}-1 \\
\mathrm{~min}\end{array}$ & $\begin{array}{c}72^{\circ} \mathrm{C}-2 \\
\text { min }\end{array}$ & $\begin{array}{c}72^{\circ} \mathrm{C}-1 \\
\text { min }\end{array}$ & $\begin{array}{c}72^{\circ} \mathrm{C}-1 \\
\mathrm{~min}\end{array}$ \\
\hline & $\begin{array}{c}72^{\circ} \mathrm{C}-5 \\
\min \end{array}$ & $\begin{array}{l}94^{\circ} \mathrm{C}- \\
1 \mathrm{~min}\end{array}$ & $\begin{array}{c}72{ }^{\circ} \mathrm{C} \\
-5 \\
\text { min }\end{array}$ & $\begin{array}{l}72{ }^{\circ} \mathrm{C}- \\
5 \mathrm{~min}\end{array}$ & $\begin{array}{c}94^{\circ} \mathrm{C}-1 \\
\mathrm{~min}\end{array}$ & $\begin{array}{l}72^{\circ} \mathrm{C}- \\
5 \mathrm{~min}\end{array}$ & $\begin{array}{c}72{ }^{\circ} \mathrm{C}-10 \\
\mathrm{~min}\end{array}$ & $\begin{array}{c}72{ }^{\circ} \mathrm{C}-10 \\
\mathrm{~min}\end{array}$ & $\begin{array}{c}72{ }^{\circ} \mathrm{C}-10 \\
\mathrm{~min}\end{array}$ \\
\hline & $\begin{array}{c}15^{\circ} \mathrm{C}- \\
\infty\end{array}$ & $\begin{array}{l}66^{\circ} \mathrm{C}- \\
1 \mathrm{~min}\end{array}$ & $\begin{array}{c}15{ }^{\circ} \mathrm{C} \\
-\infty\end{array}$ & $\begin{array}{c}15{ }^{\circ} \mathrm{C}- \\
\infty\end{array}$ & $\begin{array}{c}62^{\circ} \mathrm{C}-1 \\
\mathrm{~min}\end{array}$ & $\begin{array}{c}15^{\circ} \mathrm{C}- \\
\infty\end{array}$ & $15^{\circ} \mathrm{C}-\infty$ & $15^{\circ} \mathrm{C}-\infty$ & $15^{\circ} \mathrm{C}-\infty$ \\
\hline & & $\begin{array}{c}72^{\circ} \mathrm{C}- \\
1 \mathrm{~min} \\
30 \mathrm{seg}\end{array}$ & & & $\begin{array}{c}72^{\circ} \mathrm{C}-1 \\
\text { min } 30 \\
\text { seg }\end{array}$ & & & & \\
\hline & & $\begin{array}{c}72 \circ \mathrm{o}- \\
5 \mathrm{~min}\end{array}$ & & & $\begin{array}{c}72^{\circ} \mathrm{C}-5 \\
\mathrm{~min}\end{array}$ & & & & \\
\hline & & $\begin{array}{c}15^{\circ} \mathrm{C}- \\
\infty\end{array}$ & & & $15^{\circ} \mathrm{C}-\infty$ & & & & \\
\hline
\end{tabular}

Tabela 02 - Ciclos padronizados.

Legenda: Repete-se 30x; Repete-se 35x;

\section{DISCUSSÃO}

A HAC é a forma mais frequente dos casos de DDS e a principal causa de ambiguidade genital. A investigação de mutações derivadas do pseudogene CY21A1P por PCR-AS pode definir o diagnóstico em cerca de $90 \%$ dos casos. ${ }^{6}$

As mutações aqui investigadas estão relacionadas aos fenótipos PS, VS e à forma não clássica da HAC.

A mutação p.Pro30Leu (c.89C>T) é observada em $17 \%$ dos alelos da forma não clássica da HAC. ${ }^{15}$

A mutação c.290-13A/C>G é caracterizada pela transição dos alelos A/C para $G$ na região doadora de splicing do íntron 2, sendo esta a mutação mais frequente descrita na literatura. ${ }^{16}$ Esta alteração é responsável pela formação de uma região doadora de splicing anômala, que está associada a uma grave deficiência da CYP21A2 e ao fenótipo OS. ${ }^{16-17}$

A mutação frameshift p.Gly110fs (c.329_336delGAGACTAC) é caracterizada pela deleção de oito nucleotídeos na posição 707-714, no éxon 3 do gene CYP21A2,

Rev. Port. Saúde e Sociedade. 2019;4(1): 985 - 993. 
gerando uma enzima sem atividade funcional. Essa alteração é encontrada em 3\% a $10 \%$ dos alelos da forma OS. ${ }^{15-16}$

A mutação missense p.Ile172Asn é caracterizada pela troca de uma timina por uma adenina (c.515T>A) no aminoácido 172 da CYP21A2. Esta mutação está associada ao fenótipo VS, permitindo uma pequena produção de aldosterona, sendo suficiente para evitar as crises de perda de sal, e tem uma frequência de $5 \%$ a $10 \%$ nos alelos afetados. ${ }^{15}$

A alteração denominada Cluster6 é caracterizada por um grupo de três mutações missenses que ocorrem simultaneamente nos códons 236, 237 e 239 do éxon 6 do CYP21A2, causando a substituição de três aminoácidos - isoleucina, valina e metionina -, por asparagina, ácido glutâmico e lisina, respectivamente. Estas alterações fazem com que o gene mutado codifique uma proteína sem atividade e, sendo assim, este conjunto de mutações está associado à forma PS e possui uma frequência mundial de $3 \%$ a $17 \%$ entre todos os alelos afetados. ${ }^{6,15}$

A mutação p.Val281Leu (c.841G>T) reduz a atividade da CYP21A2 para $20 \%$ e está relacionada à forma não clássica da HAC. ${ }^{18}$

A mutação nonsene p.Gln318* (c. 952C>T) gera uma proteína truncada e com atividade nula. Essa alteração abole a função da CYP21A2 e está associada à forma PS, com frequência de $4 \%$ a $7 \%$ nos casos OS. ${ }^{15-19}$

A mutação p.Arg356Trp (c.1066C>T) gera uma enzima com, aproximadamente, 2\% de atividade, o que explica a associação à forma clássica PS. Esta mutação ocorre em 14\% dos alelos. No Brasil, a sua frequência é de $8,2 \%$ a $8,6 \%$, sendo que, no Estado de Sergipe, a frequência dessa alteração é de $14,3 \% \cdot{ }^{15,19-20}$

A abordagem molecular é fundamental para se complementar o diagnóstico clínico de HAC e, assim, fornecer o tratamento mais adequado e individualizado. A técnica PCR-AS possui a limitação de apenas indicar as mutações selecionadas. Quando as características clínicas e laboratoriais indicam a HAC, mas a alteração genética não é encontrada por meio da PCR-AS, outras técnicas são usadas para a elucidação diagnóstica, como o sequenciamento do gene CYP21A2.

\section{CONCLUSÃO}

A padronização PCR-AS para o diagnóstico molecular de HAC, devido a mutações no gene CYP21A2, no Laboratório de Genética Molecular Humana,

Rev. Port. Saúde e Sociedade. 2019;4(1): 985 - 993. 
proporcionará a elucidação diagnóstica de casos triados pelo Programa Nacional de Triagem Neonatal e/ou atendidos no Serviço de Genética Clínica do HUPAA/UFAL. Além disso, viabilizará o reconhecimento das mutações mais frequentes no gene CYP21A2 na população de Alagoas, assim como a ampliação do conhecimento sobre as relações genótipo-fenótipo para os casos de HAC. Este conhecimento é de fundamental importância para se incrementarem a abordagem diagnóstica, o tratamento, o aconselhamento genético e a prevenção.

\section{REFERENCES}

1. Marciel-Guerra AT, Guerra-Júnior G. Menino ou menina? distúrbios da diferenciação do Marciel-Guerra AT, Guerra-Júnior G. Menino ou menina? distúrbios da diferenciação do sexo. 2nd ed. Rio de Janeiro: Rubio; 2010.

2. Speiser PW, Azziz R, Baskin LS, Ghizzoni L, Hensle TW, Merke DP, et al. Congenital adrenal hyperplasia due to steroid 21-hydroxylase deficiency: an endocrine society clinical practice guideline. J clin endocrinol metab. 2010 Sept; 95(9):4133-166. Doi: $10.1210 /$ jc. $2009-2631$

3. Nimkarn S, Lin-Su K, New MI. Steroid 21 hydroxylase deficiency congenital adrenal hyperplasia. Pediatric clinics of north America. 2011 Oct; 58(5):1281-300. Doi: 10.1016/j.pcl.2011.07.012.

4. Kamp HJV, Wit JM. Neonatal screening for congenital adrenal hyperplasia. Eur $\mathrm{j}$ endocrino [Internet]. 2004 [cited 2018 Aug 15]; 151:U71-5. Available from: http://citeseerx.ist.psu.edu/viewdoc/download?doi=10.1.1.625.6853\&rep=rep1\&t ype $=$ pdf

5. Therrell BL. Newborn screening for congenital adrenal hyperplasia. Endocrinol metab clin north am [Internet]. 2001 Mar [cited 2018 Aug 10]; 30(1):15-30. Available from: https://www.ncbi.nlm.nih.gov/pubmed/11344933

6. White PC, Speiser PW. Congenital adrenal hyperplasia due to 21-hydroxylase deficiency. Endocr rev. 2000 Jun; 21(3):245-91. Doi: 10.1210/edrv.21.3.0398

7. Speiser PW, White PC. Congenital adrenal hyperplasia. The new england journal of medicine. 2003 Aug; 349(8):776-88. Doi: 10.1056/NEJMra021561

8. Bachega TASS, Madureira G, Brenlha EML, Ueti RC, Inácio $M$, Dènis $F T$, et al. Tratamento da hiperplasia supra-renal congênita por deficiência da 21-hidroxilase. Arq bras endocrinol metab. 2001 Jan/Feb; 45(1):64-72. Doi: http://dx.doi.org/10.1590/S0004-27302001000100010

9. Demirci C, Witchel FS. Congenital adrenal hyperplasia. Dermatol ther. 2008 Sept/Oct; 21(5):340-53. Doi: 10.1111/j.1529-8019.2008.00216.x.

10. Silveira EL, Santos EP, Bachega TAS, Nader VDL, Gross JL, Elnecave RH. The actual incidence of congenital adrenal hyperplasia in Brazil may not be as high as inferred - an estimate based on a public peonatal screening program in the state of Goiás. J Pediatr Endocrinol Metabol [Internet]. 2008 May [cited 2018 Aug 10]; 21(5):45560. Available from: https://www.ncbi.nlm.nih.gov/pubmed/18655527

Rev. Port. Saúde e Sociedade. 2019;4(1): 985 - 993. 
11. Pezzuti IL, Barra CB, Mantovani RN, Januário JN, Silva IN. A three-year follow-up of congenital adrenal hyperplasia newborn screening. J pediatr. 2014 Mar/Apr; 90(3):300-07. Doi: http://dx.doi.org/10.1016/j.jped.2013.09.007

12. Nascimento ML, Cristiano AN, Campos TD, Ohira M, Cechinel E, Simoni G, et al. Ten-year evaluation of a neonatal screening program for congenital adrenal hyperplasia. Arq bras endocrinol metab [Internet]. 2014 Oct [cited 2018 Aug 7]; 58(7);765-71. Available from: https://www.ncbi.nlm.nih.gov/pubmed/25372587

13. Trakakis E, Loghis C, Kassanos D. Congenital adrenal hyperplasia because of 21hydroxylase deficiency - A genetic disorder of interest to obstetricians and gynecologists. Obstet gynecol surv. 2009 Mar; 64(3):177-89. Doi: 10.1097/OGX.0b013e318193301b

14. Institute of Medical Genetics in Cardiff. The Human Gene Mutation Database. CYP21A2. [Internet]. Wales: Cardiff University; 2017 [cited 2018 Dec 15]. Available from: http://www.hgmd.cf.ac.uk/ac/gene.php?gene=CYP21A.

15. Mello MP, Bachega TASS, Costa-Santos M, Mermejo LM, Castro M. Molecular Bases of Congenital Adrenal Hyperplasia. Arq bras endocrinol metab. 2002 Aug; 46(4):457-77. Doi: http://dx.doi.org/10.1590/S0004-27302002000400017

16. Higashi $Y$, Tanae $A$, Inoue $H$, Hiromasa $T$, Fujii-Kuriyama $Y$. Aberrant splicing and missense mutations cause steroid 21-hydroxylase [P-450(C21)] deficiency in humans: possible gene conversion products. Proc Natl Acad Sci U.S.A [Internet]. 1998 Oct [cited 2018 Aug 10]; 85(20):7486-90. Available from: https://www.ncbi.nlm.nih.gov/pmc/articles/PMC282216/

17. Bachega TA, Billerbeck AE, Madureira G, Marcondes JA, Longui CA, Leite MV, et al. Molecular genotyping in brazilian patients with the classical and nonclassical forms of 21-hydroxylase deficiency. J clin endocrinol metab. 1998 Dec; 83(12):4416-19. Doi: $10.1210 /$ jcem.83.12.5350

18. Khajuria R, Walia R, Bhansali A, Prasad R. The spectrum of CYP21A2 mutations in Congenital Adrenal Hyperplasia in an Indian cohort. Clin chim acta. 2017 Jan; 464:189-94. Doi: 10.1016/j.cca.2016.11.037

19. Globerman H, Amor M, Parker KL, New MI, White PC. Nonsense mutation causing steroid 21-hydroxylase deficiency. J clin invest. 1988 July; 82:139-44. Doi: 10.1172/JCI113562

20. Campos VC, Pereira RMC, Torres N, Castro M, Aguiar-Oliveira MH. High frequency of Q318X mutation in patients with congenital adrenal hyperplasia due to 21hydroxylase deficiency in northeast Brazil. Arq Bras Endocrinol Metabol. 2009 Feb; 53(1):40-6. Doi: http://dx.doi.org/10.1590/S0004-27302009000100007

Rev. Port. Saúde e Sociedade. 2019;4(1): 985 - 993. 